\title{
Riqueza de cupins (Insecta, Isoptera) em áreas de Mata Atlântica primária e secundária do sudeste da Bahia
}

\author{
Yana T. Reis ${ }^{1,2} \&$ Eliana M. Cancello ${ }^{1}$ \\ 1. Laboratório de Isoptera, Museu de Zoologia da Universidade de São Paulo, Av. Nazaré 481, Ipiranga, 04263-000 São Paulo, SP, Brasil. \\ (ecancell@usp.br) \\ 2. Curso de Pós-Graduação em Entomologia, Faculdade de Filosofia, Ciências e Letras de Ribeirão Preto, Universidade de São Paulo, \\ 14940-605 Ribeirão Preto, SP. (yanatr@usp.br)
}

\begin{abstract}
Termite (Insecta, Isoptera) richness in primary and secondary Atlantic Forest in southeastern Bahia. The Atlantic Forest is considered one of the most diverse biome in the World, harboring a large number of endemic species. However, it is one of the most degraded ecosystems in Brazil; less than $8 \%$ of its original vegetation has been preserved. In southeastern part of the state of Bahia, irresponsible deforestation and misuse of land have being degrading the forest. In this area, the termite fauna is poorly known and there was no available survey. This paper aimed: 1) to carry out a first survey on the Isoptera from this region of the Atlantic Forest; 2) to compare the termite richness between primary and secondary forests using two kind of collecting protocols; 3 ) to compare the results from the two different collecting protocols; 4) to classify the species into trophic groups. The study areas are "Mata da Esperança" (primary forest) and "Reserva Zoobotânica da CEPLAC" (secondary forest), both in the county of Ilhéus, BA. Quantitative and qualitative surveys followed standard protocols and the samples were obtained directly, without baits. Thirty-eight species within twenty-seven genera were identified and the family Termitidae was the most frequent. The termite fauna of the primary forest was the richest. Nineteen and thirty-three species were registered with the quantitative and qualitative protocol, respectively. The majority of the species was classified as xilophagous.
\end{abstract}

KEYWORDS. Atlantic Forest, Isoptera, termite, biodiversity, richness.

RESUMO. A Mata Atlântica é considerada um dos biomas com maior diversidade do mundo, abrigando um grande número de espécies endêmicas, porém é um dos mais degradados do Brasil, restando menos de $8 \%$ de sua vegetação original. No Sudeste da Bahia, a floresta vem sofrendo um processo de deterioração, originado pelo desmatamento indiscriminado e o mau uso do solo. Nessa região, pouco se conhece sobre a fauna de cupins, onde não existia qualquer levantamento prévio. Os objetivos foram: 1) fazer uma primeira amostragem da fauna de Isoptera dessa parte da Mata Atlântica, através de duas técnicas de coleta; 2) comparar a riqueza de espécies de uma área de mata primária com outra de mata secundária; 3) comparar os resultados dos diferentes protocolos; 4) fazer classificação das espécies em grupos tróficos. As áreas de estudo foram a "Mata da Esperança" (mata primária) e a "Reserva Zoobotânica da CEPLAC" (secundária), localizadas no município de Ilhéus, BA. Foram feitas coletas chamadas aqui de "qualitativas" e "quantitativas", que seguiram protocolos determinados, e as amostras foram obtidas por coletas diretas, sem uso de iscas. Foram identificadas 38 espécies, distribuídas em 27 gêneros, sendo que a família Termitidae foi a mais freqüente. A fauna de Isoptera foi mais rica na mata primária. A partir das coletas "quantitativas", foram registradas 19 espécies, e através das coletas "qualitativas", 33 espécies. A maioria das espécies encontradas é xilófaga.

PALAVRAS-CHAVE. Mata Atlântica, Isoptera, cupins, biodiversidade, riqueza.

A Mata Atlântica tem sido alvo de maior atenção nas últimas décadas, em função da devastação que vem sofrendo. A floresta cobria inicialmente uma superfície total estimada em mais de $1.360 .000 \mathrm{~km}^{2}$, ou seja, $15 \%$ do território nacional (ALMEIDA, 2000). Atualmente o bioma está reduzido a menos de 7\% de sua extensão original, constituído por várias manchas verdes aleatoriamente distribuídas, somando $90.000 \mathrm{~km}^{2} \mathrm{e}$ ainda abriga um alto grau de diversidade de espécies e alto nível de endemismos (MORELlato \& HADDAD, 2000).

No sentido de melhorar o conhecimento da fauna de Isoptera e Hymenoptera ao longo desse bioma, foi desenvolvido um projeto temático dentro do Programa BIOTA/FAPESP: Riqueza e diversidade de Hymenoptera e Isoptera ao longo de um gradiente latitudinal na Mata Atlântica - a floresta pluvial do leste do Brasil, do qual o presente trabalho faz parte.

De modo geral, pode-se dizer que a fauna de cupins da região Neotropical é pouco conhecida, quando comparada aos cupins das regiões Oriental e Africana. Em relação aos Isoptera da Mata Atlântica, foram feitas algumas pesquisas em poucas áreas. Assim, BANDEIRA et al. (1998) estudaram a composição da fauna de cupins em João Pessoa, Paraíba, e constataram que entre $65 \%$ e $88 \%$ das espécies registradas eram novas, o que demonstra o pouco conhecimento que se tem sobre esses insetos na região. Na mesma área de trabalho, SILVA \& BANDEIRA (1999) analisaram a abundância e distribuição vertical de cupins no solo e Medeiros et al. (1999) investigaram as revoadas de térmitas. Numa compilação dos resultados dos trabalhos realizados em João Pessoa, pelo grupo de pesquisa liderado por Adelmar Bandeira, da UFPB, BANDEIRA \& VASCONCELLOS (1999) chegaram ao número total de 60 espécies. Ressaltase que esse número refere-se à soma total das espécies registradas a partir de diferentes técnicas de coleta. BRANDÃO (1998) analisou a diversidade de térmitas na Reserva Florestal de Linhares (ES) e verificou uma diminuição de riqueza com a alteração da estrutura da área.

Segundo EgGLETon et al. (1994), os cupins estão distribuídos na faixa de florestas tropicais e subtropicais, com um gradiente de aumento na riqueza de gêneros que vai das altas às baixas latitudes. 
A importância ecológica dos isópteros em ecossistemas tropicais é alta, principalmente quando consideradas as modificações que podem causar no ambiente, desde alterações de paisagem até modificações nas propriedades físicas e químicas do solo, efeitos no processo de decomposição, ciclagem de nutrientes, entre outros (LeE \& Wood, 1971; Holt \& LePAGE, 2000).

Vários autores fizeram diferentes tentativas de classificar as espécies de cupins em grupos tróficos, de acordo com a sua utilização dos recursos (MATHEws, 1977; WoOd, 1977; BANDEIRA \& MACAMBIRA, 1988; BANDEIRA, 1989; Constantino, 1992; EgGleton et al., 1995). Como o conhecimento da biologia de muitas espécies de Isoptera ainda é parco, há dificuldades para classificá-las nos diferentes grupos.

Comparações de diversidade e riqueza de cupins entre áreas de mata primária e áreas onde houve descaracterização da vegetação original foram realizadas por BANDEIRA (1989) e EGGLETON et al. (1995), com técnicas de amostragem e resultados diferentes. No trabalho de BANDEIRA (1989), a diferença entre uma área de floresta primária e outra de pastagem foi drástica. Como esperado, a fauna de mata primária foi a mais rica. No trabalho de EGGLETON et al. (1995), foram investigadas diferentes áreas, num gradiente de alteração de campos abertos, plantações recentes, áreas em processo regenerativo, mata secundária antiga e mata primária, todas em uma reserva de floresta em Mbalmayo, e a maior riqueza foi encontrada na mata secundária antiga.

Os objetivos do presente trabalho foram, além de se fazer um levantamento usando o mesmo protocolo quantitativo usado no projeto temático citado anteriormente, verificar, dentro do bioma Mata Atlântica do sudeste da Bahia, quais as alterações na riqueza de térmitas em uma área de mata primária e outra de mata secundária; verificar as diferenças dos resultados entre as coletas chamadas quantitativas e qualitativas e, por último, fazer uma classificação das espécies em grupos tróficos.

\section{MATERIAL E MÉTODOS}

As áreas estudadas, classificadas como mata primária e mata secundária, pertencem ao domínio de Mata Atlântica na região de Ilhéus, BA. A área de mata primária é conhecida como Mata da Esperança (1447'50”S, $39^{\circ} 03^{\prime} 82^{\prime}$ 'W) e trata-se de uma área remanescente de grande maciço florestal, caracterizada pela alta incidência de endemismo e pela conhecida distribuição disjunta de plantas em relação à Mata Amazônica (MEndonçA \& Amorin, 1998). A área de mata secundária pertence ao Centro de Pesquisa da Lavoura Cacaueira/CEPLAC e é conhecida como Reserva Zoobotânica (14¹8'65'S, 39॰14'74'W). É um sistema secundário, onde houve intervenção humana para o uso da terra, com finalidade agrícola, descaracterizando a vegetação primária; porém, desde 1971 (ano de tombamento), encontra-se em processo regenerativo.

As áreas estão situadas em uma zona de clima quente e úmido (tipo AF), típico de florestas tropicais sem estação seca e apresentando umidade superior a $80 \%$. O índice pluviométrico é superior a $1.800 \mathrm{~mm}$ anuais, e a temperatura média é de $24^{\circ} \mathrm{C}$ (NIMER, 1989).
As coletas foram realizadas de dezembro de 2000 a março de 2001, sempre entre 9h e 17h. A área total amostrada foi de $3.000 \mathrm{~m}^{2}$, e o esforço foi de 120 horas. Em cada tipo de mata, com o protocolo quantitativo, foi amostrada uma área de $300 \mathrm{~m}^{2}$, e através do qualitativo, uma área de $1.200 \mathrm{~m}^{2}$. O tempo gasto em cada protocolo foi de 30 horas.

Para as coletas quantitativas foram marcados seis transectos, em cada localidade, distando um do outro pelo menos $100 \mathrm{~m}$; cada um subdividido em cinco parcelas de $5 \mathrm{x}$ $2 \mathrm{~m}$, dispostas à esquerda e à direita, alternadamente e com espaçamento de $10 \mathrm{~m}$ entre elas, num total de 30 parcelas por localidade. Foi mantida também a distância de $200 \mathrm{~m}$ da borda da mata, para evitar o "efeito de borda". Os cupins foram procurados no solo, no folhiço, sob ou dentro de troncos caídos, em troncos mortos, sob a casca das árvores até a altura de 1,5 m, sob pedras, em galerias, no solo através de buracos de $15 \mathrm{~cm}$, em raízes de gramíneas ou de arbustos, ou seja, em praticamente todos os micro-habitats onde poderiam ser encontrados, anotando-se em código para posterior classificação das espécies em grupos tróficos. Como escala de tempo padrão, cada parcela foi explorada durante 1 hora/coletor.

As 30 horas gastas durante a amostragem quantitativa foram utilizadas como tempo padrão para as coletas qualitativas. Assim, para a amostragem qualitativa, em cada área (primária e secundária), seis transectos de $100 \mathrm{~m}$ x $2 \mathrm{~m}$ foram investigados num período de 5 horas/ transecto. Nesse tipo de coleta a busca só é feita em locais onde há maior probabilidade de encontrar cupins, ou seja, em ninhos visíveis (arborícolas ou epígeos) e nos micro-habitats mais conspícuos. Também alguns lotes foram coletados fora dos protocolos adotados, porém não foram considerados nas discussões.

Para as análises estatísticas, cada parcela foi considerada uma amostra independente. Os dados foram tabulados em forma de matriz de presença/ausência. Essa matriz também foi utilizada para a construção das curvas de riqueza acumulada, através do programa "EstimateS" (versão 5.0).

Para comparar as áreas em termos de riqueza, foi utilizado o estimador de Jackknife de $1^{\mathrm{a}}$ ordem, que normalmente é utilizado para comparações entre diferentes áreas com o mesmo esforço amostral. O uso de um estimador é importante porque não é possível fazer um censo total da comunidade, apenas uma estimativa. Através dele foi obtida uma estimativa do número esperado do total de espécies para cada área (HELTSHE \& FoRRESTER, 1983). Para verificar a suficiência amostral foram feitas curvas de acumulação de espécies nas duas áreas, utilizando-se apenas os dados das coletas quantitativas.

O hábito alimentar dos cupins foi estudado por observação direta no campo, além de se considerar os dados da literatura. A classificação dos térmitas em grupos tróficos foi feita de acordo com o trabalho de EGGLETON et al. (1995): humívoros ou geófagos (cupins que se alimentam de solo); intermediários, conhecidos como "soil/wood interface-feeders" (cupins que se alimentam da interface solo/madeira, são coletados predominantemente no solo imediatamente sob troncos caídos ou colados a eles, ou ainda, dentro de troncos em alto estágio de decomposição, onde o solo está misturado com madeira muito degradada); xilófagos (cupins que se 
alimentam de madeira); e ceifadores ou comedores de serrapilheira (cupins que cortam folhas ou alimentam-se de pequenos fragmentos de madeira e/ou outros itens da serrapilheira).

As referências bibliográficas dos trabalhos de descrições de espécies aqui citadas estão em ConstanTino (1998). Todo o material coletado encontra-se depositado no Museu de Zoologia da Universidade de São Paulo.

\section{RESULTADOS}

Foram coletados 325 lotes de cupins, identificados (por EMC) como 38 espécies em 27 gêneros. Dessas espécies, 22 foram consideradas como ainda não descritas (Tab. I). Algumas amostras de Syntermes dirus (Burmeister, 1839) (Termitidae: Nasutitermitinae) foram encontradas ocasionalmente, fora dos protocolos

Tabela I. Espécies de Isoptera registradas em mata primária e secundária, no município de Ilhéus, BA, durante o período de dezembro de 2000 e janeiro de 2001, através de amostragens qualitativa e quantitativa (MP, mata primária; MS, mata secundária; QT, amostragem quantitativa; QL, amostragem qualitativa).

\begin{tabular}{|c|c|c|c|c|c|c|}
\hline \multirow[t]{3}{*}{ Espécies } & \multicolumn{4}{|c|}{ Freqüência Absoluta } & \multirow[t]{3}{*}{ Grupos Tróficos } & \multirow{3}{*}{$\begin{array}{l}\mathrm{N}^{\mathrm{o}} \text { total de } \\
\text { registros }\end{array}$} \\
\hline & \multicolumn{2}{|c|}{ MP } & \multicolumn{2}{|c|}{ MS } & & \\
\hline & QT & QL & QT & QL & & \\
\hline \multicolumn{7}{|l|}{ KALOTERMITIDAE } \\
\hline Cryptotermes sp. & & 2 & & & Xilófagos & 2 \\
\hline Calcaritermes rioensis Krishna, 1962 & 3 & 2 & & 2 & Xilófagos & 7 \\
\hline Eucryptotermes sp. & & 1 & & & Xilófagos & 1 \\
\hline Rugitermes rugosus (Hagen, 1858) & & & & 1 & Xilófagos & 1 \\
\hline \multicolumn{7}{|l|}{ RHINOTERMITIDAE } \\
\hline Dolichorhinotermes sp. & 3 & 2 & & & Xilófagos & 5 \\
\hline Heterotermes sp. 1 & 1 & 3 & & 2 & Xilófagos & 6 \\
\hline Heterotermes sp. 2 & & & & 4 & Xilófagos & 4 \\
\hline Rhinotermes sp. & & 1 & & & Xilófagos & 1 \\
\hline \multicolumn{7}{|l|}{ TERMITIDAE } \\
\hline \multicolumn{7}{|l|}{ Apicotermitinae } \\
\hline Anoplotermes sp. 1 & 5 & 1 & 3 & & Humívoros & 9 \\
\hline Anoplotermes sp. 2 & 1 & & & & Humívoros & 1 \\
\hline Anoplotermes sp. 3 & 2 & & & & Humívoros & 2 \\
\hline Tetimatermes sp. & & & 1 & & Humívoros & 1 \\
\hline \multicolumn{7}{|l|}{ Nasutitermitinae } \\
\hline Angularitermes pinocchio Cancello \& Brandão, 1996 & 1 & 1 & & & Humívoros & 2 \\
\hline Agnathotermes sp. & & 3 & & & Humívoros & 3 \\
\hline Araujotermes sp. & & 2 & & & Humívoros & 2 \\
\hline Atlantitermes sp. 1 & & 1 & & & Humívoros & 1 \\
\hline Atlantitermes sp. 2 & & 1 & & & Humívoros & 1 \\
\hline Armitermes euamignathus Silvestri, 1901 & 4 & 5 & 2 & 6 & Xilófagos & 17 \\
\hline Convexitermes manni (Emerson, 1925) & 1 & & & & Intermediários & 1 \\
\hline Diversitermes sp. & & 5 & & 1 & Xilófagos & 6 \\
\hline Embiratermes neotenicus (Holmgren, 1906) & & 11 & & & Intermediários & 11 \\
\hline Ereymatermes sp. & & 1 & & & Humívoros & 1 \\
\hline Labiotermes labralis (Holmgren, 1906) & 1 & 3 & & & Humívoros & 4 \\
\hline Nasutitermes sp. 1 & & 2 & & & Xilófagos & 2 \\
\hline Nasutitermes sp. 2 & & & & 35 & Xilófagos & 35 \\
\hline Nasutitermes callimorphus Mathews, 1997 & & & & 1 & Xilófagos & 1 \\
\hline Nasutitermes corniger (Motschulsky, 1855) & 4 & 8 & 12 & 61 & Xilófagos & 85 \\
\hline Nasutitermes ephratae (Holmgren, 1910) & 1 & 6 & 8 & 19 & Xilófagos & 34 \\
\hline Nasutitermes jaraguae (Holmgren, 1910) & 5 & & & & Xilófagos & 5 \\
\hline Nasutitermes macrocephalus (Silvestri, 1903) & & & 1 & 1 & Xilófagos & 2 \\
\hline Nasutitermes minor (Holmgren, 1906) & 5 & 3 & 1 & & Xilófagos & 9 \\
\hline Subulitermes sp. & & 4 & & & Humívoros & 4 \\
\hline Velocitermes sp. & & & & 2 & Ceifadores & 2 \\
\hline \multicolumn{7}{|l|}{ Termitinae } \\
\hline Amitermes sp. & 2 & 8 & 1 & 14 & Xilófagos & 25 \\
\hline Cavitermes tuberosus (Emerson, 1925) & 1 & 5 & & & Humívoros & 6 \\
\hline Crepititermes verruculosus (Emerson, 1925) & & 1 & & & Humívoros & 1 \\
\hline Orthognathotermes sp. & & 1 & & & Humívoros & 1 \\
\hline Termes sp. & 2 & 15 & & 7 & Intermediário & 24 \\
\hline Total geral de registros & 42 & 98 & 29 & 156 & & 325 \\
\hline Número de espécies & 17 & 27 & 08 & 14 & & 38 \\
\hline
\end{tabular}


adotados, portanto, a soma total de espécies é 39. Na mata primária foram registradas 31 espécies, sendo 21 exclusivas dessa área, enquanto que na mata secundária foram registradas apenas 17 , sendo seis exclusivas dessa área (Tab. I).

As três famílias encontradas foram, como esperado, Kalotermitidae, Rhinotermitidae e Termitidae, a última representando $73 \%$ das espécies amostradas. Aproximadamente $45 \%$ das espécies registradas pertencem a Nasutitermitinae (Termitidae), sendo que oito delas são do gênero Nasutitermes Dudley, 1890.

Através da amostragem quantitativa, foi encontrado um total de 19 espécies, das quais cinco foram registradas exclusivamente através desse método. A partir das coletas qualitativas, foi encontrado um total de 33 espécies, sendo 17 registradas exclusivamente através desse método. E ainda, 14 espécies foram registradas através dos dois métodos de coletas (Tab. I).

Considerando-se apenas as coletas quantitativas, na mata primária, o número de espécies observado foi de 17 e o estimado, através do Jackknife de $1^{\mathrm{a}}$ ordem, de 25 , enquanto na mata secundária, o observado foi de 8 e o estimado de 12.

A partir das curvas de acumulação de espécies (Figs. 1,2), verifica-se que o número de espécies observado não está próximo do estimado, pois foi capturado apenas $66 \%$ da fauna esperada. Estatisticamente, o esforço amostral é considerado satisfatório quando, através do protocolo adotado, é possível capturar pelo menos $85 \%$ da riqueza esperada; portanto, o esforço amostral não foi suficiente para coletar a maior parte da fauna de cupins existente nas duas áreas.

Em relação aos grupos tróficos na mata primária, foram encontradas 14 espécies xilófagas, 14 humívoras e três intermediárias (Fig. 3). Na mata secundária, foram encontradas 12 espécies xilófagas, duas humívoras, uma intermediária e uma ceifadora.

\section{DISCUSSÃO}

Os resultados do presente trabalho corroboram os obtidos por BANDEIRA (1989) quanto à maior riqueza na mata primária, apesar do método utilizado ter sido outro e a diferença entre as áreas, naquele caso, ter sido muito mais drástica. BANDEIRA (1989) concluiu que alterações microclimáticas podem modificar a composição faunística, pois certas espécies de cupins não suportam dessecamento e grandes flutuações de temperatura. Entretanto, tais fatores não seriam explicações para as diferenças encontradas nas duas matas de Ilhéus, uma vez que a mata secundária não difere da primária tanto quanto uma pastagem de uma mata primária.

EGGLETON et al. (1995) constataram que há um aumento considerável de riqueza nas áreas classificadas como floresta secundária antiga (área sem alteração por 30 anos) e plantações recentes em relação à floresta primária. Segundo os autores, as áreas secundárias antigas funcionariam como grandes clareiras artificiais, com dossel fechado e abundante suprimento de madeira, deixada pela própria perturbação original. No caso do presente trabalho, não é possível utilizar o mesmo raciocínio, pois foi encontrada maior quantidade de troncos caídos na

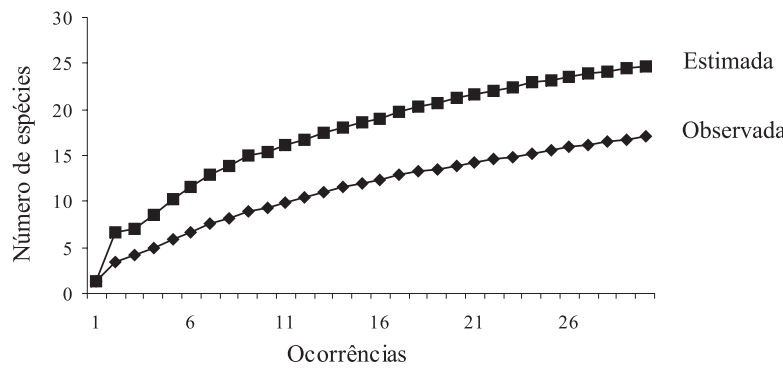

Fig. 1. Curvas estimada e observada de acumulação de espécies de Isoptera na mata primária, no município de Ilhéus, BA, em 2000.

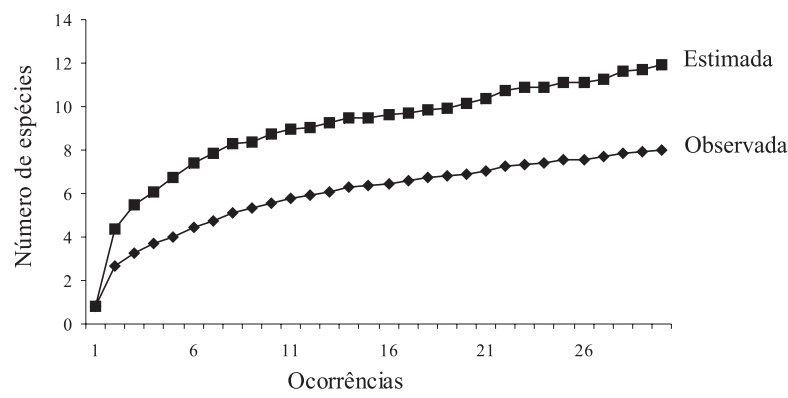

Fig. 2. Curvas estimada e observada de acumulação de espécies de Isoptera, obtidas através do Jackknife de $1^{\text {a }}$ ordem, na mata secundária, no município de Ilhéus, BA, em 2001.

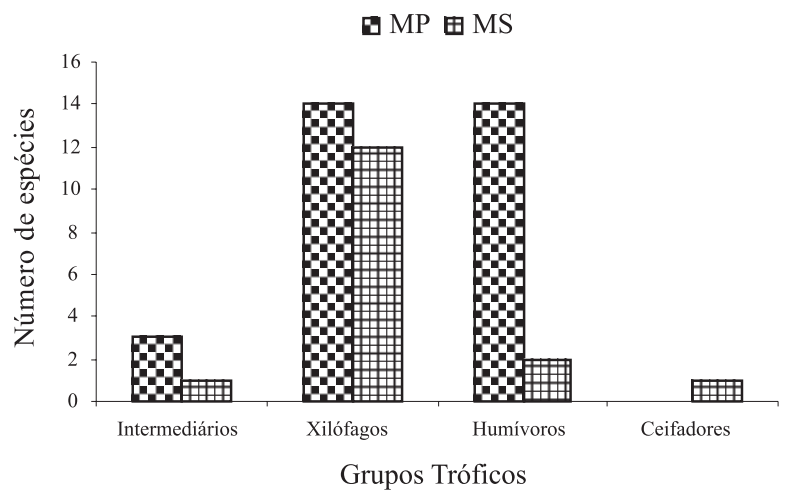

Fig. 3. Distribuição dos grupos tróficos de Isoptera existentes na mata secundária (MS) e primária (MP) no município de Ilhéus, BA, 2000-2001

mata primária do que na secundária. Como não foi feita nenhuma análise mais cuidadosa envolvendo parâmetros como estrutura da vegetação, composição do solo e/ou abundância de madeira morta, analisada de forma quantitativa, não se pode afirmar que os resultados obtidos sejam ou não conseqüência de tais fatores.

Na mata secundária, observou-se alta frequiência de Nasutitermes corniger, considerada uma espécie xilófaga comum e de grande importância econômica na região Nordeste, onde é uma das pragas urbanas mais freqüientes (A. Bandeira, com. pess.). Segundo Constantino (2002), $N$. corniger é uma das mais importantes espécies-praga desse gênero na América do Sul. Como quase toda espécie-praga, esta apresenta certa plasticidade, inclusive quanto aos ninhos, que podem ser policálicos, aumentando a área de 
forrageamento de cada colônia. BANDEIRA et al. (2003) relatam que esta espécie não foi registrada em floresta primária e foi a mais freqüente em áreas onde houve algum tipo de distúrbio. Embiratermes neotenicus ocorreu em alta freqüência na mata primária e não foi encontrada na mata secundária (Tab. I). Os indivíduos foram encontrados em ninhos no solo, em troncos em estágio avançado de decomposição, em contato com o solo. Resultado semelhante foi encontrado por BANDEIRA et al. (2003), que afirmaram ser a espécie típica e abundante nas Florestas Amazônica e Atlântica, sendo encontrada apenas em áreas primárias ou primárias perturbadas. Esses insetos podem se alimentar da interface solo/madeira, mas é difícil afirmar os motivos da diferença de sua ocorrência entre as duas matas. Uma única espécie de Amitermes, determinada como ainda não descrita, foi registrada em ambas as áreas (Tab. I). Os exemplares dessa espécie foram encontrados em diversos locais, como troncos caídos em diferentes estados de decomposição ou em árvores vivas ou mortas, às vezes em perfeito estado de conservação (neste caso, ocorrendo sob a casca da árvore). Também foram encontrados muitas vezes junto a outras espécies num mesmo micro-habitat, porém em partes distintas. A espécie pode explorar a celulose em diferentes formas, madeira em diferentes estágios de decomposição e daí a alta frequiência nos dois tipos de mata. Comparando-se com material da coleção, foi possível verificar que ela ocorre também em alta freqüência em locais geograficamente próximos, mas com diferentes coberturas vegetais, desde outros tipos de florestas, como em áreas abertas, de caatingas.

A riqueza de Isoptera, quando comparada a de outros grupos de insetos, como formigas, por exemplo, pode ser considerada baixa (Delabie et al., 2000). Entretanto, mesmo sabendo que o esforço de coleta foi relativamente pequeno, analisando-se os resultados de levantamentos faunísticos de térmitas em florestas brasileiras, verifica-se que o número aqui encontrado não foi muito diferente do que já foi publicado. Constantino (1992) registrou 90 espécies (64 em Maraã e 26 perto de Belém) num total de $7.500 \mathrm{~m}^{2}$ de área em florestas de terra firme na Amazônia, ou seja, duas vezes e meia maior do que a investigada no presente trabalho. Assim, pode-se dizer que, proporcionalmente, o número encontrado em Ilhéus, de 37 espécies, é compatível com o encontrado na Amazônia. É difícil fazer uma comparação mais aprofundada entre as espécies dos dois trabalhos, pois além de diferentes esforços de coleta e técnicas empregadas, são muitas as morfoespécies, consideradas como ainda não descritas (Tab. I). Entre as espécies nomeadas (Tab. I), apenas quatro constam entre as enumeradas por Constantino (1992): Labiotermes labralis, Nasutitermes corniger, Cavitermes tuberosus e Crepititermes verruculosus.

Um estudo realizado por BANDEIRA et al. (1998), em duas reservas de Mata Atlântica de João Pessoa, (PB), somando $4.000 \mathrm{~m}^{2}$ foram encontradas 43 espécies. Comparando-se com o número de espécies de Ilhéus, de novo, a proporção é semelhante. Também é difícil comparar as listas de espécies de BANDEIRA et al. (1998) com a da Tabela I do presente trabalho, pois em ambos os casos as listas contém muitas morfoespécies. Aqueles autores afirmam que $67 \%$ do total é constituído de espécies que ainda não foram descritas. Quanto aos gêneros, fora os de Apicotermitinae, apenas Microcerotermes (Termitidae, Termitinae) e Coptotermes (Rhinotermitidae), ambos xilófagos, foram registrados lá e não em Ilhéus, BA. Nasutitermes foi o gênero mais freqüente e o mais diversificado em todos estes estudos. Em ambos os levantamentos de Mata Atlântica, a maioria das espécies é xilófaga.

É necessário discutir ainda os limites advindos da técnica empregada, ou seja, um tipo de levantamento rápido, com um esforço de coleta relativamente pequeno, coleta direta (sem iscas), apenas em uma época do ano e sempre durante o dia (entre 9 h e 17h). Há grupos taxonômicos e/ou grupos tróficos subestimados e castas pouco representadas (alados). A baixa freqüência do grupo ceifador, por exemplo, pode ter sido ocasionada pelo horário da coleta, pois esse grupo costuma forragear ao anoitecer e/ou à noite. Um outro exemplo é a baixa freqüência dos Kalotermitidae, que certamente foram subestimados, pois como vivem sempre dentro de madeira e, muitas vezes, madeira bem dura, ainda pouco degradada, é difícil amostrá-los em levantamentos rápidos, fato já registrado na literatura (CANCELLO, 1996). As curvas de acumulação de espécies mostram que o esforço amostral foi insuficiente para coletar a maior parte da fauna. Assim, seria prematuro aceitar como definitivo o resultado mostrado na Figura 3 sobre as proporções dos grupos tróficos. Talvez com maior esforço de coleta e com variação nos horários, por exemplo, outros grupos fossem melhor representados.

JoNES \& EGGLETON (2000) realizaram um trabalho para verificar a eficiência de um tipo de protocolo de coleta semelhante ao aqui utilizado (quantitativo), a partir de comparações com os resultados de levantamentos anteriores mais abrangentes e verificaram ser possível amostrar de $31 \%$ a $36 \%$ da fauna conhecida. Segundo aqueles autores, com o protocolo quantitativo seria possível amostrar uma área maior em um período menor, permitindo que os dados fossem recolhidos mais rapidamente, sem perda significativa da eficácia. Porém, o que foi verificado no presente trabalho levanta dúvidas quanto a estas afirmações, uma vez que $50 \%$ do total de espécies registrado em Ilhéus foi encontrado exclusivamente através da amostragem qualitativa.

Através da análise dos resultados aqui apresentados, pode-se afirmar que, para levantamentos faunísticos mais abrangentes, com intenção de inventariamento da fauna, é importante diversificar os tipos de amostragem, pois as diferentes técnicas de coleta podem revelar faunas um pouco diferentes. Seria ainda necessário, no caso de inventários mais completos, utilizar não só os dois tipos de coletas aqui mencionados, mas também procurar diversificar horário de coleta, épocas diferentes e usar diferentes tipos de iscas, por exemplo. Percebe-se também que a falta de uma metodologia unificada nos diversos trabalhos já realizados no Brasil dificulta a comparação dos resultados conseguidos por diferentes pesquisadores. Neste sentido, sugerimos mais discussões entre a comunidade para verificar qual seria a melhor técnica a ser empregada quando se pretende fazer levantamentos faunísticos rápidos.

Apesar de terem sido registradas 23 espécies 
consideradas como não descritas, ainda não se pode considerá-las endêmicas. Espera-se que, com a análise total das espécies das outras 14 áreas do projeto temático citado anteriormente (do Programa BIOTA/FAPESP), seja possível responder a esta e a outras questões importantes, mas já se pode afirmar que algumas destas espécies registradas em Ilhéus, BA, também foram encontradas em outras localidades do projeto temático.

Ainda são necessários mais estudos, como da estrutura das mandíbulas e do tubo digestivo dos operários de muitas espécies, de conteúdo do tubo digestivo dos operários (DoNOvAn et al., 2001) e, principalmente, observações cuidadosas e diretas no campo, para que se possa confirmar a dieta dos indivíduos de muitas espécies.

Apesar das dificuldades da classificação das espécies em grupos tróficos, é importante esta tentativa para melhor compreender o papel de cada grupo nas comunidades e assim obter parâmetros melhores para utilização desses organismos como bioindicadores.

Agradecimentos. À Fundação de Amparo a Pesquisa do Estado de São Paulo (FAPESP-Proc. $n^{\circ}$ 00/03160-9), pelo financiamento da bolsa de mestrado de YTR e do projeto temático do Programa Biota/FaPesP (Proc. 9805083-0) e, conseqüentemente do presente trabalho e ao CNPq, pela bolsa de Produtividade em Pesquisa (nº 30333304/87-1) de EMC. A Jacques H. C. DeLabie (Universidade Estadual de Santa Cruz/UESC) pela leitura crítica do texto. A Christian Bordereau, pela orientação e ajuda à YTR no período de coleta. A Marcelo Duarte (Museu de Zoologia/ USP) pela leitura crítica do abstract. A Reginaldo Constantino (Universidade de Brasília), pela identificação de parte dos Nasutitermes e aos amigos Rogério Silva, Antonio Tavares, Ivan Cardoso e Nelson Florencio Filho pela preciosa contribuição na construção das planilhas, nos cálculos estatísticos e nas coletas. Também aos revisores anônimos da Revista que auxiliaram com importantes correções e sugestões.

\section{REFERÊNCIAS BIBLIOGRÁFICAS}

Almeida, D. S. DE. 2000. Recuperação ambiental da Mata Atlântica. Ilhéus, Editus - Universidade Estadual de Santa Cruz. 130p.

BANDEIRA, A. G. 1989. Análise da termitofauna (Insecta: Isoptera) de uma floresta primária e de uma pastagem na Amazônia Oriental, Brasil. Boletim do Museu Paraense Emílio Goeldi, Série Zoologia, 5:225-241.

Bandeira, A. G. \& Macambira, M. L. J. 1988. Térmitas da Carajás, Estado do Pará, Brasil: composição faunística, distribuição e hábito alimentar. Boletim do Museu Paraense Emílio Goeldi, Série Zoologia, 4(2):175-190.

Bandeira, A. G. \& Vasconcellos, A. 1999. Estado atual do conhecimento sistemático e ecológico sobre os cupins (Insecta, Isoptera) do nordeste brasileiro. Revista Nordestina de Biologia 13(1/2):37-45.

Bandeira, A. G.; Pereira, J. C. D.; Miranda, Y. C. S. \& Medeiros, L. G. S. 1998. Composição da fauna de cupins (Insecta: Isoptera) em áreas de Mata Atlântica em João Pessoa, Paraíba, Brasil. Revista Nordestina de Biologia 12(1/2):9-17.

Bandeira, A. G.; Vasconcellos, A.; Silva, M. P. \& Constantino, R. 2003. Effects of habitat disturbance on the termite fauna in a highland humid forest in the Caatinga domain, Brazil. Sociobiology 42(1):117-127.

BRANDÃO, D. 1998. Patterns of the termite (Isoptera) diversity in the Reserva Florestal de Linhares, State of Espírito Santo, Brazil. Revista Brasileira de Entomologia 41(2-4):151-153.

Cancello, E. M. 1996. Termite diversity and richness in Brazil an overview. In: Bicudo, C. E. DE M. \& Menezes, N. A. eds. Biodiversity in Brazil - a first approach. São Paulo, CNPq. p.173-182.

Constantino, R. 1992. Abundance and diversity of Termite (Insecta: Isoptera) in two sites of primary rain forest in Brazilian Amazonia. Biotropica 24(3):420-430.

1998. Catalog of the termites of the New World (Insecta:Isoptera). Arquivos de Zoologia 35(2):135-230.

2002. The pest termites of South America: taxonomy, distribuition and status. Journal of Applied Entomolgy 126:355-365

Delabie, J. H. C.; Agosti, K. \& Nascimento, I. C. 2000. Litter ant communities of the Brazilian Atlantic rain Forest region. In: Agosti, D.; Majer, J.; Alonso, L. \& Schults, T. eds. Sampling ground-dwelling ants: case studies from the world's rain forest. Perth, Curtin University, School of Environmental Biology. p.1-17. (Bulletin, 18).

Donovan, S.; EgGleton, P. \& Bignell, D. E. 2001. Gut content analysis and a new feeding group classification of termites. Ecological Entomology 26:356-366.

EgGleton, P.; Williams, P. H. \& Gaston, K. 1994. Explaining global diversity: productivity or history? Biodiversity and Conservation 3:318-330.

Eggleton, P.; Bignel, D. E.; Sands, W. A.; Waite, B.; Wood, T. G. \& Lawton, J. H. 1995. The species richness (Isoptera) under differing levels of forest disturbance in the Mbalmayo Forest Reserve, southern Camerron. Journal of Tropical Ecology 11:85-98.

Heltshe, J. F. \& Forrester, N. E. 1983. Estimating species richness using the jackknife procedure. Biometrics 39:1-11.

Holt, J. A. \& Lepage, M. 2000. Termites and soil properties. In Abe, T.; Bignell, D. E. \& Higashi, M. eds. Termites, evolution, sociality, symbiosis, ecology. Dordrecht, Kluwer Academic. p.389-407.

Jones, D. T. \& Eggleton, P. 2000. Sampling termite assemblages in tropical forests: testing a rapid biodiversity assessment protocol. Journal of Applied Ecology 37:191-203.

Lee, K. E. \& Wood, T. G. 1971. Termites and soil. London, Academic. 251p.

Mathews, A. G. A. 1977. Studies on termites from the Mato Grosso State, Brazil. Rio de Janeiro, Academia Brasileira de Ciências. 267p

Medeiros, L. G. S.; Bandeira, A. G. \& Martius, C. 1999. Termite swarming in the Nottheastern Atlantic Rain Forest of Brazil. Studies on Neotropical Fauna and Environment 34:76-87.

Mendonça, R. \& Amorin, A. 1998. Reserva Biológica de Una Itabuna, CEPEC/CEPLAC. 30p. (Boletim Informativo, 7).

Morellato, L. P. C. \& Haddad, C. F. B. 2000. Introduction: the Brazilian Atlantic Forest. Biotropica 32(4b):786-792.

Nimer, E. 1989. Climatologia do Brasil. Rio de Janeiro, IBGE. $422 p$.

Silva, E. G. \& Bandeira, A. G. 1999. Abundância e distribuição vertical de cupins (Insecta, Isoptera) em solo de Mata Atlântica, João Pessoa, Paraíba. Revista Nordestina de Biologia 13(1/2):13-36.

Wood, T. G. 1977. The effects of clearing and grazing on the termite fauna (Isoptera) of tropical savanas and Woodlands. In: VAneK, J. ed. Progress in soil Zoology. Prague, Academia. p.409-418.

Recebido em julho de 2006. Aceito em novembro de 2006. ISSN 0073-4721

Artigo disponível em: www.scielo.br/isz 